\title{
Afecto, besos y condones: el $A B C$ de las prácticas sexuales de las trabajadoras sexuales de la Ciudad de México
}

Betania Allen, M en Antropol, ${ }^{(1)}$ Aurelio Cruz-Valdez, M en C, Dr en C, ${ }^{(1)}$ Leonor Rivera-Rivera, M en C, ${ }^{(1)}$ Roberto Castro, Dr en Sociol, ${ }^{(2)}$ María Ernestina Arana-García, M en Formación D ocente, DEA en Educación, ${ }^{(3)}$ Mauricio Hernández-Avila, MC, D r en C.(1)

\section{Allen B, Cruz-Valdez A, Rivera-Rivera L, Castro R, Arana-García ME, Hernández-Avila M. Afecto, besos y condones: el ABC de las prácticas sexuales de las trabajadoras sexuales de la Ciudad de México. Salud Publica Mex 2003;45 supl 5:S594-S607. El texto completo en inglés de este artículo está disponible en: http://www.insp.mx/salud/index.html}

\section{Resumen}

Objetivo. D escribir las prácticas sexuales de las trabajadoras sexuales de la Ciudad de México y explorar qué significan para ellas. Material y métodos Se trata de un estudio de abordaje cualitativo y cuantitativo llevado a cabo,el primero, en 1992 y, el segundo, de 1991 a 1992. U na muestra representativa de trabajadoras sexuales $(n=335)$ de un marco muestral respondió a un cuestionario y 52 de ellas participaron en seis grupos focales. Se calcularon frecuencias simples, medidas de tendencia central y de dispersión. Los datos cualitativos se interpretaron a la luz de la teoría fundamentada y la perspectiva feminista. Resultados Con clientes las trabajadoras sexuales aceptan la penetración vaginal y (menos) la oral, mientras rechazan la penetración anal y los besos. Con parejas personales todas las prácticas sexuales son aceptadas. En su vida profesional las trabajadoras sexuales usan la disociación psico-corporal como una estrategia de defensa psicológica y para construir su identidad. Los servicios solicitados por el último cliente fueron: $98.4 \%$ vaginal, $39.1 \%$ oral, $7.7 \%$ anal y se reportó uso del condón con $94 \%$. Con la pareja personal, $72.6 \%$ de las trabajadoras sexuales reportaron nunca usar el condón. La semana anterior al estudio las trabajadoras sexuales laboraron, en promedio, $3.7 \pm 1.5$ días y tuvieron relaciones

\author{
Allen B, Cruz-Valdez A, Rivera-Rivera L, Castro R, \\ Arana-García ME, Hernández-Avila M. \\ Affection, kisses and condoms:The basics of sexual \\ practices of female sex workers in Mexico City. \\ Salud Publica Mex 2003;45 suppl 5:S594-S607. \\ The English version of this paper \\ is available at: http://www.insp.mx/salud/index.html
}

\begin{abstract}
A bstract
Objective.To describe and explore the meanings of sexual practices among female sex workers (FSW ) in Mexico City. Material and Methods.A qualitative and quantitative study was car ried out.The qualitative approach was conducted in 1992 and the latter from 1991 to 1992.The study population was a representative sample of FSW $(n=335)$ who completed a questionnaire; 52 of them also participated in six focal groups. Simple frequencies, central trend, and dispersion measures were calculated. $Q$ ualitative data were interpreted using G rounded Theory and a feminist perspective. Results. Female sex workers accepted vaginal penetration with clients and oral penetration to a lesser extent, while anal penetration and kissing were rejected.All sexual modalities were accepted with per sonal partners. In their practice, FSW use psychophysical dissociation as a psychological defense mechanism and to construct their identities. Services requested by their latest clients were: $98.4 \%$ vaginal, $39.1 \%$ oral, and $7.7 \%$ anal sex. Condom use was reported by $94 \%$. W ith their personal partner, $72.6 \%$ of FSW never used condoms. The week before the study, FSW worked $3.7 \pm 1.5$ days on average and had sexual intercourse with $8.6 \pm 8.1$ clients. Conclusions Interventions are needed to promote safe sex among FSW in their private and professional lives.
\end{abstract}

Esta investigación fue posible gracias al financiamiento proporcionado por la Fundación Rockefeller, la Fundación A mericana para la Investigación sobre el SIDA (AmFAR) y la Red de SIDA y Salud Reproductiva (ARHN).

(1) Centro de Investigación en Salud Poblacional, Instituto N acional de Salud Pública.

(2) Centro Regional de Investigaciones Multidisciplinarias, Universidad N acional Autónoma de México, Cuernavaca, Mor, México.

(3) Coordinación Académico-Administrativa del Instituto de Ciencias de la Educación, Universidad Autónoma del Estado de Morelos, Cuernavaca, Morelos, México.

Fecha de recibido: 15 de febrero de 2002 • Fecha de aprobado: 18 de septiembre de 2003 Solicitud de sobretiros: Maestra Betania Allen. 7ạ Cerrada de Fray Pedro de Gante 50, colonia Sección 16, Delegación Tlalpan, 14000 México, D F, México. Correo electrónico: ballen@ correo.insp.mx 
sexuales con $8.6 / \pm 8.1$ clientes. Conclusiones. Es necesario diseñar intervenciones que promuevan el sexo seguro entre trabajadoras sexuales en sus vidas privadas y profesionales. El texto completo en inglés de este artículo está disponible en: http://www.insp.mx/salud/index.html

Palabras clave: trabajadoras sexuales; uso del condón; prevención del VIH; prácticas sexuales; prácticas de riesgo paraVIH; México
The English version of this paper is available at: http:// www.insp.mx/salud/index.html

Key words: female sex workers; condom use; HIV prevention; sexual practices; HIV risk practices; M exico
$H$ ay muchas más investigaciones sobre las trabajadoras del comercio sexual que sobre mujeres con otros tipos de vulnerabilidad al VIH/SIDA, ${ }^{1,2}$ en parte porque al principio de la epidemia del SIDA se pensó que ellas podrían constituir un grupo de alto riesgo para la transmisión del VIH/SIDA. Esto se debió en cierta medida a que algunos estudios reportaron altas prevalencias de infección por el VIH entre trabajadoras sexuales en Africa, y entre aquellas mujeres que además de hacer trabajo sexual usaban drogas intravenosas. Muchos autores extrapolaron este hallazgo a las trabajadoras sexuales en general, y a veces lo hicieron sin estudiar sus prácticas riesgosas específicas. ${ }^{3}$ Además, en la mayoría de los artículos sobre el trabajo sexual femenino y el VIH/SIDA subyace una concepción de las trabajadoras sexuales como una categoría de personas que suelen tener enfermedades y ser un peligro para la "población general". ${ }^{4}$ Estas concepciones estigmatizan a las mujeres que hacen trabajo sexual, relegándolas a "grupos de riesgo" en vez de promover la investigación sobre sus prácticas de riesgo específicas y los factores que pudieran influir en éstas. Es decir, muchas investigaciones sobre trabajadoras sexuales parecen asignarle mayor importancia y valor al riesgo que corre la "población general" que a las vidas de las trabajadoras sexuales. ${ }^{5}$ En lugar de esto, habría que investigar las realidades de las mujeres que hacen trabajo sexual para poder apoyarlas con intervenciones efectivas, ya que se ha visto que las trabajadoras sexuales son especialmente vulnerables al VIH/ SIDA dadas sus frecuentes desventajas económicas y por estar en posiciones de menor poder, lo cual las puede orillar a escoger entre la supervivencia económica e inmediata y la protección contra el VIH/SIDA. ${ }^{6}$

Estudios rigurosos sobre este tema (lo cual implica, generalmente, que fueron hechos desde una perspectiva de género) han mostrado que las trabajadoras sexuales constituyen un grupo heterogéneo en cuanto a su ambiente laboral, situación socioeconómica, salud (estado y patrones de atención), prácticas sexuales y conocimiento y uso de medidas de prevención de la transmisión del VIH. ${ }^{3,7}$ Además, varía la manera en que conciben sus vidas privadas y profesionales, y el nivel de riesgo de las prácticas que tienen en cada esfera vivencial. Por lo tanto, las tasas de seroprevalencia de VIH entre trabajadoras sexuales varían de un país a otro, y de una región a otra en un solo país. ${ }^{7}$ El riesgo de infección por el VIH de las trabajadoras sexuales parece depender, más que del número de parejas sexuales que tengan, de tres factores generales: la existencia de un alto nivel de prevalencia de VIH/SIDA en la población general, como ocurre en algunas regiones de Africa; el uso de drogas intravenosas por ellas y sus parejas sexuales, y las prácticas sexuales específicas en las cuales participan, ya sean riesgosas o seguras. $^{7-9}$

Por otro lado, algunos autores plantean que hay tres situaciones que pueden contribuir a una baja prevalencia del VIH entre las trabajadoras sexuales: a) una prevalencia baja del VIH entre la población general; b) una distribución desigual de la prevalencia del VIH en la población, combinada con una tendencia entre las personas infectadas a no tener contacto sexual con las trabajadoras sexuales, y c) que las prácticas sexuales de estas trabajadoras no promuevan la transmisión del VIH. ${ }^{10}$

El último factor en los dos casos -las prácticas sexuales riesgosas o seguras de las trabajadoras sexuales- podría ser el más importante en México, debido al bajo nivel de uso de drogas intravenosas entre trabajadoras sexuales (al menos en la Ciudad de México) así como la baja prevalencia del VIH entre la población general. ${ }^{10,11} \mathrm{Al}$ parecer, estos factores han contribuido a las bajas prevalencias de infección por el VIH entre trabajadoras sexuales mexicanas. ${ }^{10-12}$

Las prácticas sexuales de las mujeres que hacen trabajo sexual a su vez se ven afectadas por cuestiones sociales de gran complejidad: el poder desigual que tienen la mujer y el hombre en la negociación sexual, $8,13,14$ el tipo de sitio en el cual trabajan y la manera en que está organizada la administración que allí existe, $^{3,5,12,15,16}$ el nivel de solidaridad entre las trabaja- 
doras sexuales en un sitio de trabajo, ${ }^{17,18}$ el conocimiento que ellas tengan sobre el sexo más seguro, ${ }^{3,7,9}$ y la pobreza. ${ }^{13,14}$ Por lo tanto, la promoción del sexo más seguro y la prevención de la transmisión del VIH entre trabajadoras sexuales es, como se ha visto en otras poblaciones, un problema complejo que requiere de investigación antes de poder proponer soluciones efectivas. Además, diversos estudios indican que la vigilancia de la prevalencia de VIH/SIDA entre trabajadoras sexuales y la investigación sobre los factores de riesgo en esta población es importante para países con epidemias de VIH/SIDA que son concentradas, como es el caso de México. ${ }^{10,19}$

Los datos y el análisis aquí presentados forman parte de una investigación hecha durante los años 90 que busca producir estrategias efectivas de prevención de la transmisión del VIH/SIDA entre las trabajadoras sexuales y sus clientes y parejas personales en la Ciudad de México. También se pretende contribuir al conocimiento general de la sexualidad con la descripción de datos empíricos en relación con un grupo social específico. ${ }^{20}$ Este artículo describe las prácticas sexuales (incluyendo el sexo más seguro) de un grupo de trabajadoras sexuales de la Ciudad de México y explora los significados que estas prácticas tienen para ellas, puesto que pensamos que sólo se puede entender la sexualidad si comprendemos los significados que la construyen. ${ }^{21,22}$

\section{Material y métodos}

\section{Población de estudio}

Para este artículo, nuestra población de estudio son las trabajadoras sexuales de sitios de trabajo ubicados en las calles de la Ciudad de México. En el componente cualitativo del estudio se incluyeron 52 trabajadoras sexuales, mientras en la parte cuantitativa se estudiaron 335. Todas las participantes trabajaban en sitios laborales de calle y eran de dos niveles socioeconómicos: medio y bajo.* Se escogió estudiar este grupo de

\footnotetext{
* Dentro del componente cualitativo del estudio, estos niveles socioeconómicos se definieron a partir de una estimación del costo de los servicios que ofrecen las trabajadoras sexuales, con base en información recolectada con observación etnográfica. En esta investigación, en 1990-1991, se clasificó como "bajo" a los sitios con precios de N\$20 a N\$40 y como "medio-bajo" (equivalente al nivel "medio" del componente cuantitativo) de N\$41 a N\$100. En el componente cuantitativo del estudio, los niveles socioeconómicos se definieron con base en el índice de Bronfman y colaboradores, que combina la escolaridad, tipo de vivienda y servicios, y bienes materiales. ${ }^{23}$
}

trabajadoras sexuales (las que trabajan en sitios de calle) porque algunos estudios han indicado que son las que tienen más riesgo de infección por el VIH. ${ }^{24,25}$

\section{Métodos cualitativos}

El análisis cualitativo se hizo entre 1991 a 1992, antes del cuantitativo, que tuvo lugar en 1992. Los datos cualitativos se recolectaron a través de seis grupos focales con 52 trabajadoras sexuales. Una psicóloga con experiencia en el método moderó las entrevistas grupales con base en una guía con módulos temáticos. Después de recibir por escrito el consentimiento informado de las participantes, se grabaron las entrevistas para su posterior captura. Las entrevistas grupales se codificaron con base en categorías definidas por una antropóloga y un sociólogo. Para sistematizar esta información se utilizó un programa de cómputo para el análisis cualitativo (Tally), y para analizarla, la teoría fundamentada y la perspectiva feminista. ${ }^{26-31}$

Se buscó, a través del análisis cualitativo, conocer y describir las prácticas sexuales entre las trabajadoras sexuales estudiadas y sus clientes o parejas privadas. Además, intentamos entender, a través de la interpretación, cómo ellas le dan significado a estas prácticas y cómo las experimentan. Se propone que algo tan íntimo y complejo como las prácticas sexuales, sean éstas profesionales o privadas, sólo puede entenderse cabalmente si las ubicamos dentro de la estructura de significados con la cual las trabajadoras sexuales las entienden.

\section{Métodos cuantitativos}

Para el componente cuantitativo del estudio se desarrolló un marco muestral; la unidad primaria de muestreo fueron los sitios donde se ejerce el trabajo sexual (bares, calles y estéticas) de una zona de la Ciudad de México. La información para la construcción del marco muestral fue obtenida de informantes clave, incluyendo trabajadoras sexuales, taxistas, meseros, administradoras de sitios de trabajo sexual y personal del entonces llamado Consejo Nacional para la Prevención del SIDA (CONASIDA).

El marco muestral se plasmó en un mapa que identifica los sitios de sexo comercial, que especifica ubicación geográfica y datos como el tipo de sitio y el número aproximado de trabajadoras. De este mapa se seleccionaron de manera aleatoria 20 sitios de calle, 30 de bar y 10 de estética, los cuales conformaron el marco muestral. Con base en este marco se seleccionó una muestra aleatoria representativa de trabajadoras sexuales; el tamaño de muestra fue de 826 participantes. Una descripción detallada de la construcción de este mar- 
co muestral aparece en otra publicación. ${ }^{24}$ Se construyó el marco muestral para intentar reducir el sesgo de selección que es frecuente en estudios con trabajadoras sexuales. ${ }^{18}$ Para fines del análisis presentado en el presente artículo se seleccionaron los datos de las 335 mujeres que trabajaban en sitios de calle.

Entre esta población de estudio se aplicó un cuestionario estructurado que incluía preguntas acerca de características sociodemográficas, estado de salud, prácticas sexuales profesionales y privadas, conocimientos, percepción de riesgo y prácticas con relación a VIH/SIDA/infecciones de transmisión sexual (ITS). Los cuestionarios fueron aplicados por 12 entrevistadores (cuatro mujeres y ocho hombres) con experiencia en la aplicación de cuestionarios y en el trabajo de campo dentro de estudios sobre el VIH/SIDA y las ITS que involucran a trabajadoras sexuales como población de estudio.

Se capturó la información en una base de datos en el programa DBASE/IV. En primera instancia, se analizó la información recolectada con los cuestionarios a través de la medición de variables continuas, lo que arrojó frecuencias simples, medidas de tendencia central y de dispersión. La distribución de cada variable se graficó para observar la hipótesis de normalidad y se realizó una prueba de comparación, cuando fue necesaria, entre grupos con y sin el evento bajo estudio. De acuerdo con el comportamiento de las variables continuas, éstas fueron categorizadas. La medición del evento de interés se hizo a través de la prevalencia del uso de condón, tanto en clientes como en parejas privadas.

\section{Resultados}

\section{Características sociodemográficas}

La media de edad de las trabajadoras sexuales que participaron en el componente cuantitativo del estudio fue de 28 años (desviación estándar DS=9.30). Respecto al estado civil, más de la mitad $(66.6 \%, n=223)$ de las trabajadoras sexuales eran solteras y casi la tercera parte $(29.9 \%, n=100)$ eran divorciadas, separadas o viudas, y sólo $3.6 \%(n=12)$ mencionaron estar casadas actualmente.

Aproximadamente la mitad $(51.9 \%, n=174)$ de las trabajadoras sexuales que completaron el cuestionario eran de nivel socioeconómico bajo, y la otra mitad de nivel medio (48.1\%, $n=161)$. En cuanto a la educación, $42.5 \%(n=142)$ se clasificó en un nivel medio (carrera técnica o secundaria incompleta o completa) y $51.8 \%$ $(n=173)$ se ubicó en un bajo nivel educativo (no fue a la escuela, primaria incompleta o completa) (cuadro I).

\section{Prácticas sexuales entre trabajadoras sexuales y clientes}

Frecuencia de las prácticas sexuales con clientes

En promedio, las trabajadoras sexuales trabajaron 3.7 días (DS=1.51) durante la semana anterior a la aplicación del cuestionario. Las trabajadoras sexuales tuvieron relaciones sexuales con un promedio de 8.6 clientes durante la semana anterior al estudio (DS=8.12). Ellas tuvieron, en promedio, 2.1 relaciones sexuales con clien-

\section{Cuadro I \\ Datos SOCIODEMOgRÁficos \\ DE TRABAJADORAS SEXUALES DE SITIOS DE CALLE de La CiUdad de MéXICO, 1991-1992*}

Variable Número \%

Edad

Media $=28$ años (DS=9.30)

\begin{tabular}{lrr} 
16-22 años & 105 & 31.4 \\
\hline 23-27 años & 91 & 27.2 \\
\hline 28-32 años & 61 & 18.3 \\
\hline 33-37 años & 31 & 9.3 \\
\hline >38 años & 46 & 13.8
\end{tabular}

Estado civil

\begin{tabular}{lrr} 
Casada & 12 & 3.6 \\
\hline Soltera & 223 & 66.6 \\
\hline 0 tro & 100 & 29.9
\end{tabular}

$\mathrm{N}$ ivel socioeconómico

\begin{tabular}{lll} 
Medio & 161 & 48.1 \\
\hline Bajo & 174 & 51.9
\end{tabular}

Escolaridad

\begin{tabular}{lrr} 
Alta & 19 & 5.7 \\
\hline Mediana & 142 & 42.5 \\
\hline Baja & 173 & 51.8
\end{tabular}

Indice de educación y nivel socioeconómico

\begin{tabular}{lrr} 
Alto & 14 & 4.2 \\
\hline Mediano & 82 & 24.6 \\
\hline Bajo & 138 & 71.3
\end{tabular}

* Todos los cuadros se refieren a los datos de las trabajadoras sexuales de sitios de calle de la Ciudad de México que completaron el cuestionario cuantitativo. El número total de trabajadoras de sitios de calle fue de 335; suman menos de 335 cuando hubo valores missing

$n=335$ 
tes al día (DS=1.65). La gran mayoría de las trabajadoras sexuales de sitios laborales en la calle tienen pocos clientes con quienes no tienen relaciones sexuales, ya que $92.2 \%(n=307)$ dijeron que siempre tienen relaciones con sus clientes

En general, las trabajadoras sexuales dijeron que pasan una cantidad de tiempo extremadamente limitado con sus clientes: $42.4 \%$ ( $n=141)$ pasó 10 minutos o menos con su último cliente, y 30.7\% $(n=102)$ pasó entre 11 y 15 minutos (cuadro II). Esto confirma la información recopilada con métodos cualitativos, la cual indica que el servicio sexual no rebasa 15 minutos si no se recibe un pago extra.

\section{Prácticas sexuales con clientes: aceptación y rechazo}

Dentro de la actividad profesional de las trabajadoras sexuales hay varios elementos importantes que se observaron durante los grupos focales, como son las prácticas aceptadas y rechazadas con clientes y la concepción que ellas tienen de esta parte de su vida sexual. En primer lugar está la manera en la que las trabajadoras sexuales de calle organizan la prestación de sus servicios. En la Ciudad de México, además de limitar el tiempo permitido para cada cliente, las trabajadoras sexuales determinan que el precio cubre únicamente lo que ellas definen como el servicio sexual básico, que es la penetración vaginal en la posición tradicional. Ellas cobran una cantidad adicional por lo que se concibe como servicios extras, como el desnudarse, dejarse tocar, diferentes posiciones o tipos de penetración. Por ejemplo, una trabajadora sexual lo expresó así,

"Lo normal es nada más bajarse en este caso la falda o el pantalón, si quiere desnudo, se cobra aparte, la postura aparte." (GF 10)*

En cuanto a las prácticas sexuales aceptadas, en los grupos focales las participantes mantienen que la más común es la penetración vaginal. También según las respuestas de las participantes al cuestionario cuantitativo, la práctica sexual más solicitada por el último cliente anterior a la aplicación del cuestionario fue la penetración vaginal $(98.4 \%, n=300)$. $\ddagger$ También se acep-

\footnotetext{
* Por razones de confidencialidad nos referimos a los grupos focales por número, de GF 1 a GF 14.

‡ En el cuestionario, se preguntó cuáles servicios había solicitado el último cliente en vez de los que la trabajadora sexual había proporcionado porque en la prueba piloto del cuestionario se encontró que había rechazo de parte de las trabajadoras sexuales a la pregunta más directa. Los porcentajes suman más de 100 porque la trabajadora puede haber proporcionado más de un tipo de servicio, como por ejemplo penetración vaginal y plática.
}

\section{Cuadro II \\ Frecuencia del trabajo sexual entre trabajadoras SeXuales de sitios de calle de la Ciudad DE MÉxıCO, 1991-1992}

Variable

Número

$\%$

$N$ úmero de días trabajados en la semana pasada

Media $=3.7$ días (DS=1.51)

\begin{tabular}{lrr}
1 día & 13 & 4.9 \\
\hline 2 días & 44 & 16.7 \\
\hline 3 días & 74 & 28.0 \\
\hline 4 días & 48 & 18.2 \\
\hline 5 días & 30 & 11.4 \\
\hline 6 días & 55 & 20.8
\end{tabular}

$N$ úmero de clientes en la semana pasada

Media $=8.6$ clientes $(\mathrm{DS}=8.12$ )

\begin{tabular}{lrr}
$<5$ clientes & 129 & 38.5 \\
\hline $6-10$ clientes & 106 & 31.6 \\
\hline $11-16$ clientes & 32 & 9.6 \\
\hline$>17$ clientes & 68 & 20.3
\end{tabular}

N úmero de clientes "regulares" en la semana pasada

\begin{tabular}{rrr}
$<5$ & 173 & 51.6 \\
\hline $6-10$ & 26 & 7.8 \\
\hline$>11$ & 136 & 40.6
\end{tabular}

Frecuencia con la cual tiene relaciones sexuales con clientes

\begin{tabular}{lrr} 
Siempre & 307 & 92.2 \\
\hline Algunas veces & 25 & 7.5 \\
\hline N unca & 1 & 0.3
\end{tabular}

Tiempo (en minutos) que pasó con el último cliente

\begin{tabular}{lrr}
$<10$ minutos & 141 & 42.47 \\
\hline De 11-15 minutos & 102 & 30.73 \\
\hline De $16-20$ minutos & 36 & 10.85 \\
\hline De 21-30 minutos & 38 & 11.45 \\
\hline De 31-59 minutos & 3 & 0.90 \\
\hline Una hora & 8 & 2.40 \\
\hline Dos horas & 3 & 0.90 \\
\hline Tres horas & 1 & 0.30
\end{tabular}

N úmero de clientes en una "buena" semana laboral

\begin{tabular}{lrr}
$<5$ clientes & 67 & 20.0 \\
\hline $6-15$ clientes & 154 & 46.0 \\
\hline $16-20$ clientes & 42 & 12.5 \\
\hline $21-25$ clientes & 19 & 5.7 \\
\hline 26 clientes & 53 & 15.8
\end{tabular}

N úmero de clientes en una "mala" semana laboral

\begin{tabular}{lrr}
$<5$ clientes & 167 & 49.9 \\
\hline $6-10$ clientes & 59 & 17.6 \\
\hline $11-15$ clientes & 21 & 6.3 \\
\hline $16-20$ clientes & 5 & 1.5 \\
\hline 21 clientes & 83 & 24.8 \\
& &
\end{tabular}

$n=335$ 
ta, como se mencionó, el desnudarse, dejarse tocar o tocar al cliente, y en algunos casos el sexo oral. Las prácticas que se aceptan muy poco son las que las trabajadoras conciben como "antinaturales", especialmente la penetración anal. La práctica que nunca se acepta es el beso en la boca con los clientes. La siguiente cita de un grupo focal se refiere a este aspecto:

"Son muy diferentes los trabajos de diferentes lugares, nosotras no mamamos, ni beso, ni que nos estén mordiendo...porque [el cliente] le está pagando a uno por encuerarse, por ponerse uno de postura, pero nada más. Pero que lo quieran besar a uno no, o sea yo en mi persona no me dejo besar." (GF 10)

Por otra parte, casi la tercera parte de las trabajadoras sexuales $(29.1 \%, n=48)$ refirieron que el cliente había solicitado el sexo oral. La conversación o "plática" fue un servicio importante también, con $22.6 \%$ $(n=31)$. Las solicitudes para el sexo anal fueron relativamente pocas, pero alcanzaron $8.3 \%(n=11)$. Finalmente, algunos clientes solicitaron la masturbación manual $(6.1 \%, n=8)$ y el masaje $(4.7 \%, n=6)$ (cuadro III).

\section{Disociación corporal con los clientes}

Uno de los hallazgos más importantes del análisis cualitativo fue que las trabajadoras sexuales de la Ciudad de México utilizan conscientemente una barrera simbólica, emocional y sensorial, entre sí mismas y los clientes durante sus relaciones sexuales.

\section{Cuadro III \\ Prácticas sexuales solicitadas POR LOS CLIENTES, SEGÚN LO REPORTADO POR TRABAJADORAS SEXUALES DE SITIOS DE CALLE de la Ciudad de México, 1991-1992}

\begin{tabular}{lrr} 
Práctica sexual & Número & \multicolumn{1}{c}{$\%$} \\
Penetración vaginal & 300 & $98.4 \%$ \\
\hline Sexo oral & 48 & $29.1 \%$ \\
\hline Penetración anal & 11 & $8.3 \%$ \\
\hline Masturbación (manual) & 8 & $6.1 \%$ \\
\hline Plática & 31 & $22.6 \%$ \\
\hline Masaje & 6 & $4.7 \%$
\end{tabular}

* Los porcentajes suman más de 100 porque la trabajadora puede haber proporcionado más de un tipo de servicio, como por ejemplo penetración vaginal y plática

$n=335$
"Yo me bloqueo por completo cuando trabajo." (GF 10) "Es que lo hace uno en plan de trabajo. Se pone uno fría, ni piensa uno en hacerlo. Entonces uno nomás se mueve y eso, ellos terminan, se levanta uno, se lava y se va." (GF 10)

"Se aprende. Todo es mental. Sí, se acostumbra uno a no pensar en lo que está haciendo. Haga de cuenta que es uno nada más como una máquina. 'A lo que te traje y vámonos,' ¿verdad? Claro que ya con su pareja, ya es otra cosa, ¿verdad? Porque siente uno con amor a esa persona y siente uno con confianza de explayar." (GF 8)

Esta disociación corporal es una separación social y psicológica entre la persona interior y su profesión. Ellas utilizan esta barrera como una defensa frente a la violencia física y simbólica, la opresión y el estigma que en repetidas ocasiones durante las entrevistas grupales dijeron experimentar al trabajar en el comercio sexual.

"'Prefiere estar paradita que irse a trabajar a otro lado,' me han dicho. Pero no es fácil." (GF 8).

"Yo creo que si eres una mujer de la calle que se vende, no tenemos respeto en la calle." (GF 13)

"Sí hay personas que pasan, que van con su esposo, y como ellas se sienten seguras, a uno lo ven como una basura. Así nos consideran, como una lacra, como lo peor. Pero yo quisiera que por un minuto se pudieran analizar...qué necesidad tiene la mujer prostituta para hacer lo que hace. Pero nunca lo han pensado, porque las personas que lo tienen todo, nunca se ponen a pensar en las que no tienen, y qué es lo que necesitan." (GF 11)

Como resultado de la disociación, las trabajadoras perciben y viven las prácticas sexuales con sus clientes de forma diferente que con sus parejas personales. Asimismo, esto tiene influencia en su percepción del riesgo de infección del VIH y su uso del condón en sus diferentes contactos sexuales, ya sean privados o profesionales.

El ejemplo más importante de la disociación es el rechazo tajante por parte de las trabajadoras sexuales al beso en la boca con los clientes. Eso parece denotar un rechazo a la intimidad con el cliente. El rechazo al beso en la boca es mucho mayor que el rechazo que existe para algunas otras prácticas, incluyendo el sexo anal.

"Es por demás que uno se deje besar porque... para mí es repugnante." (GF 8) 
"Me dijo el cliente, 'bésame,' y le digo, 'pues no, no te puedo besar porque te acabo de conocer,' pues yo no los beso." (GF 8)

"Porque ahí no va uno a abrazarse o besarse, no. ...Sí [a los clientes] les gusta [el beso], pero yo creo que también ya saben, ¿no? Porque ya saben que siempre ha sido así. Yo creo desde que empezó, siempre ha sido así." (GF 12)

Por otra parte, las trabajadoras sexuales reportan poca frecuencia de la penetración anal con los clientes, y ellas explicaron que esto también se debe por un lado al rechazo a la intimidad con ellos, y por otro, a problemas físicos que ellas dijeron acompañan a este tipo de penetración. Sin embargo, en algunos casos las trabajadoras aceptan la posibilidad (motivada por necesidad económica) de este tipo de penetración, pero nunca la de permitir un beso en la boca.

Como se mencionó, el desnudarse, las caricias y el sexo oral tienen un costo extra; pareciera ser que se cobra más por cada elemento que simboliza una mayor intimidad entre la trabajadora y el cliente. Las trabajadoras sexuales explican la diferencia en los costos de los servicios diciendo explícitamente que prefieren no hacer estas prácticas porque tienen una mayor carga afectiva.

Otra manifestación importante de esta disociación es el rechazo a la experimentación del placer sexual y orgasmos en sus relaciones sexuales profesionales.

"La verdad, muchos clientes piden que sienta uno. Entonces uno les da por su lado, 'No, que la verdad, sí,' y finge uno. Yo al menos finjo que he llegado [al orgasmo], pero la verdad no. Yo la verdad nunca he llegado." (GF 10)

"Una mujer no se viene con los clientes, porque no." (GF 13)

"Es que nosotros venimos por necesidad y por ganar unos centavos, no es para gozarla con ellos, disfrutarlos." (GF 13)

En las discusiones grupales, las trabajadoras sexuales aceptaron que era posible experimentar excitación sexual y orgasmos con clientes, pero que esto era extremadamente raro para ellas. Cuando algunas participantes recordaron algún cliente específico con el cual habían sentido placer sexual, dijeron que lo mejor era dejar de aceptar a esa persona como cliente.

“Una vez me tocó un chavo que sí me clavé mucho. Me clavé porque llegué yo al orgasmo con él...y lo tuve que cortar...dejándolo de ver...y me volvía otra vez a blo- quear, o sea me bloqueé por completo otra vez." (GF 10)

Ellas explicaron la falta de sensaciones placenteras por el uso consciente de una barrera simbólica (ellas la llaman "bloquearse", "ponerse fría") y porque "no siente uno nada hacia esa persona" o porque "falta afecto".

\section{Sexo más seguro con los clientes}

Durante los grupos focales y en el cuestionario, la gran mayoría de las trabajadoras sexuales indicaron que utilizaban condones en todos sus contactos sexuales profesionales. Sin embargo, en los grupos focales encontramos contradicciones en su discurso que indican que no usan condones tan consistentemente como relatan en primera instancia. Pareciera que hay un uso elevado, pero no constante.

Las trabajadoras sexuales son generalmente las que proporcionan el condón para su uso en las relaciones sexuales con el cliente. Otra práctica de sexo más seguro que dicen utilizar las trabajadoras es rechazar aquellos clientes en quienes, al revisar sus genitales antes de la relación sexual, encuentren signos de ITS conocidos por ellas. Después de esta revisión, ellas son quienes les ponen el condón al cliente, aunque después de terminada la relación sexual, ellas prefieren que el cliente se quite el condón por sí mismo. Finalmente, después de la relación sexual suelen lavar su propia área genital en el baño del cuarto de hotel.

Parece generalizado entre las trabajadoras sexuales el conocimiento del condón como un método eficaz de prevención de la transmisión del VIH, y también de otras infecciones de transmisión sexual. No obstante, entre muchas de las entrevistadas, se observó una falta de información correcta sobre la necesidad de usar únicamente lubricantes a base de agua con los condones. Algunas trabajadoras sexuales sabían qué lubricantes se deben usar para prevenir la ruptura de los condones, pero indicaron que les es difícil encontrarlos.

La mayoría de las trabajadoras sexuales que completaron el cuestionario refirieron haber usado un condón con el último cliente $(97.9 \%, n=323)$. En poco menos de la mitad de los casos $(42.8 \%, n=142)$, la trabajadora sexual dijo ser quien proporciona los condones, y sólo 4.2\% (n=14) refirió que el cliente los proporciona. Puede ser un dato importante para el desarrollo de una intervención en favor del uso del condón que casi la tercera parte de las trabajadoras sexuales (31\%, $n=103$ ) reportaron que el hotel donde prestan sus servicios proporciona los condones, y 15.1\% $(n=50)$ dijo 
que la administración del sitio de trabajo les provee de condones.

Mientras $12.7 \%(n=42)$ de las trabajadoras sexuales dijeron que no hablaron con el cliente sobre el uso del condón, sólo 2.1\% ( $n=7)$ refirió no haber usado condón con el cliente. En más de la mitad de los casos $(68.1 \%, n=226)$, cuando hablaron del condón fue ella quien lo sugirió. También, 7.8\% $(n=26)$ dijeron que el cliente sugirió su uso, aunque sólo 4.2\% $(n=14)$ de ellos proporcionaron el condón (cuadro IV).

\section{Prácticas sexuales con parejas personales}

En cuanto a los antecedentes de vida sexual de las trabajadoras estudiadas, la media de la edad al iniciar la vida sexual fue de 16.5 (DS=2.75). La media de parejas regulares antes de empezar a trabajar en el comercio sexual fue de 1.2 ( $\mathrm{DS}=0.92$ ), mientras después de haber empezado a trabajar en esta profesión la media fue de 1.45 (DS=1.58). En el momento del estudio, más de la mitad de las trabajadoras sexuales a quienes se les

\section{Cuadro IV \\ USO DEL CONDÓN EN LOS CONTACTOS SEXUALES PROFESIONALES, SEGÚN LO REPORTADO \\ POR TRABAJADORAS SEXUALES DE SITIOS DE CALLE de la CiUdad de México, 1991-1992}

\begin{tabular}{lrr} 
Variable & Número & \multicolumn{1}{c}{$\%$} \\
$\begin{array}{lrr}\text { El cliente usó condón } \\
\text { Sí }\end{array}$ & & \\
\hline No & 323 & 97.9 \\
\hline
\end{tabular}

Si hay condones disponibles en el lugar de trabajo, y quién los proporciona

\begin{tabular}{lrr} 
No & 4 & 1.2 \\
\hline La trabajadora sexual & 142 & 42.8 \\
\hline El cliente & 14 & 4.2 \\
\hline La administración del sitio de trabajo sexual & 50 & 15.1 \\
\hline La administración del hotel & 103 & 31.0 \\
\hline O tro & 19 & 5.7
\end{tabular}

Si la trabajadora y el cliente hablaron acerca del uso del condón, y quién lo sugirió

\begin{tabular}{lrr} 
No hablaron & 42 & 12.7 \\
\hline Ella lo sugirió & 226 & 68.1 \\
\hline El cliente lo sugirió & 26 & 7.8 \\
\hline La administración del sitio de trabajo lo sugirió & 25 & 7.5 \\
\hline La administración del hotel lo sugirió & 13 & 3.9 \\
$n=335$ & &
\end{tabular}

aplicó el cuestionario $(68.1 \%, n=218)$ refirieron que no tenían una pareja personal (novio, esposo, pareja con quien vive, amante), mientras $30.9 \%(n=99)$ tenían una pareja y sólo $0.9 \%(n=3)$ tenían dos parejas personales (media $=0.33 ; \mathrm{DS}=0.49$ ). De las trabajadoras que tenían

\section{Cuadro V \\ Datos SObRe La VIDA SEXUAL PERSONAL \\ DE TRABAJADORAS SEXUALES DE SITIOS DE CALLE DE LA CiUdad de México, 1991-1992}

Variable

Número $\quad \%$

Edad al inicio de relaciones sexuales

Media $=16.5$ años (DS=2.752)

\begin{tabular}{lrr}
$11-15$ años & 124 & 37.7 \\
\hline $16-20$ años & 183 & 55.6 \\
\hline$>21$ años & 22 & 6.7
\end{tabular}

$N$ úmero de parejas regulares antes de empezar

a trabajar en el comercio sexual

Media=1.233 (DS=0.915)

\begin{tabular}{lrr}
1 pareja & 217 & 93.5 \\
\hline $2-4$ parejas & 10 & 4.3 \\
\hline $5-6$ parejas & 5 & 2.1
\end{tabular}

$N$ úmero de parejas regulares después de que empezó a trabajar en el comercio sexual Media $=1.45$ (DS=1.576)

\begin{tabular}{lrr}
1 pareja & 109 & 90.8 \\
\hline $2-4$ parejas & 4 & 3.3 \\
\hline $5-10$ parejas & 7 & 5.8
\end{tabular}

$N$ úmero de parejas personales actualmente Media $=0.328(\mathrm{DS}=0.49)$

\begin{tabular}{lrr} 
No tiene pareja personal & 218 & 68.1 \\
\hline Una pareja & 99 & 30.9 \\
\hline Dos parejas & 3 & 0.9
\end{tabular}

¿Cohabita con su pareja personal?

\begin{tabular}{lll} 
Sí & 61 & 58.1 \\
\hline No & 44 & 41.9
\end{tabular}

¿U sa condón con su pareja personal?*

\begin{tabular}{lrr} 
Siempre & 8 & 7.62 \\
\hline Casi siempre & 4 & 3.81 \\
\hline A veces & 16 & 15.24 \\
\hline Rara vez & 4 & 3.81 \\
\hline N unca & 73 & 69.52
\end{tabular}

* Hubo 230 valores missing para esta pregunta

$n=335$ 
una pareja, 58.1\% (n=61) vive con él (cuadro V). Estos datos nos indican que las trabajadoras sexuales viven su vida sexual privada con un número reducido de parejas.

En cuanto a las prácticas sexuales aceptadas, la vida privada de las trabajadoras sexuales es casi opuesta a su experiencia profesional. En las entrevistas grupales, las participantes dijeron que aceptaban por lo general cualquier práctica sexual con su pareja personal. La única práctica sexual que ocasionalmente es rechazada con la pareja es la penetración anal.

En especial, en su vida personal ellas ponen mucho énfasis en las caricias, los abrazos y los besos. Además, las trabajadoras sexuales dijeron que experimentaban placer durante las relaciones sexuales con sus parejas, y que tenían orgasmos con frecuencia.

Por otra parte, muchas de las trabajadoras sexuales que participaron en las discusiones grupales dijeron que rechazan el uso del condón en las relaciones sexuales privadas porque simbolizaría una barrera inaceptable entre ellas y sus parejas. En sus respuestas al cuestionario cuantitativo sólo $7.6 \%(n=8)$ de las trabajadoras sexuales dijeron siempre usar condón con su pareja personal. Explicaron que no usan el condón con su pareja personal porque implicaría una falta de confianza. También pareciera que el no usarlo es una manera de diferenciar su sexualidad privada de la profesional.

\section{Discusión}

\section{Frecuencia de las prácticas sexuales con clientes}

Como hemos indicado, las trabajadoras sexuales son un grupo heterogéneo en el ámbito mundial; los factores de riesgo específicos para la transmisión y las tasas de seroprevalencia del VIH entre ellas varían de manera pronunciada de un país a otro, e inclusive de una ciudad a otra. ${ }^{7}$ Específicamente, las prácticas de las trabajadoras sexuales en diferentes contextos sociales pueden ser muy diversas. Al comparar los resultados de nuestro análisis con los de otros estudios en diferentes países, encontramos tanto diferencias como similitudes en las prácticas sexuales de las trabajadoras sexuales estudiadas.

Un estudio realizado por Wilson y colaboradores en Bulawayo, Zimbabwe, con 113 trabajadoras sexuales, encontró que en promedio trabajaban 3.6 noches por semana, y $85 \%$ dijeron que usualmente tenían un sólo cliente por noche. ${ }^{32}$ En otro estudio de Wilson y colaboradores, entre 100 trabajadoras sexuales y 100 clientes en Harare, Zimbabwe, los autores encontra- ron que las trabajadoras sexuales laboraban 4.6 noches por semana en promedio, con 2.2 clientes por noche. ${ }^{17}$ En un estudio realizado entre 62 trabajadoras de 22 burdeles en Colombia, 79\% tenían 2-3 clientes por noche en una "buena" noche. ${ }^{33}$ En un estudio en Bali, Indonesia, con 80 trabajadoras sexuales que trabajaban con clientes que eran turistas y 100 clientes de este tipo, las trabajadoras reportaron un promedio de 3.3 clientes en la semana pasada. ${ }^{34}$ En general, la frecuencia de contactos sexuales profesionales entre trabajadoras sexuales encontrada en los estudios de Zimbabwe y Colombia fue semejante a la que encontramos en nuestro estudio (3.7 días por semana y 2.2 clientes al día), mientras la frecuencia hallada en Indonesia fue menor. Una diferencia entre las trabajadoras sexuales de la Ciudad de México y las de Bali, Zimbabwe y Colombia es que las segundas pasaban más tiempo (usualmente toda la noche) con los clientes que en nuestro estudio.

Por otro lado, trabajadoras sexuales estudiadas en Glasgow, Escocia y Camden, Estados Unidos de América (EUA), tenían una mayor frecuencia de contactos sexuales profesionales que la observada en la Ciudad de México. Por ejemplo, 63 trabajadoras sexuales de sitios de calle de la ciudad de Glasgow, Escocia, trabajaron una media de 5.5 noches por semana y tenían contacto con una media de 6.4 clientes por jornada. ${ }^{35}$ En otro estudio en Glasgow, las 68 trabajadoras sexuales estudiadas laboraban en promedio 5.2 días por semana. ${ }^{36}$ El estudio en la ciudad de Camden, (EUA), reportó que las 20 trabajadoras sexuales de sitios de calle estudiadas tenían un promedio de 4.13 clientes por día de trabajo. ${ }^{37}$ En general, estas trabajadoras sexuales europeas y estadounidenses tuvieron más clientes por noche, pero pasaron un periodo de tiempo por servicio semejante a lo que observamos en la Ciudad de México (15-20 minutos).

\section{Prácticas sexuales profesionales}

Entre las trabajadoras sexuales de Harare, Zimbabwe, que participaron en el estudio de Wilson y colaboradores, la práctica sexual más frecuente fue la penetración vaginal $(97 \%)$. También fueron frecuentes la estimulación manual de los genitales de la trabajadora (81\%) y de los del cliente (76\%), y el beso (58\%), cosa que no ocurre en las trabajadoras sexuales de la Ciudad de México. ${ }^{17}$

En un estudio en Gambia, que duró 14 meses e incluyó zonas urbanas y semirrurales, los autores decidieron que no era necesario registrar las prácticas de las trabajadoras sexuales con sus clientes, ya que de la observación etnográfica concluyeron que casi siempre el 
servicio consistía en la penetración vaginal únicamente, sin estimulación manual ni otros tipos de penetración. ${ }^{38}$

Entre la población estudiada por de Gallo y Alzate en Colombia, el servicio incluía sólo penetración vaginal; los clientes pedían (pero las trabajadoras sexuales dijeron no aceptar) servicios orales y anales. ${ }^{33}$ La diferencia más importante entre el estudio realizado en Zimbabwe y el nuestro es la aceptación del beso. Con relación a las demás prácticas sexuales profesionales, se parecen las que se aceptan en Zimbabwe y Colombia a las que nuestra población de estudio dijo aceptar.

En contraste, los grupos de trabajadoras sexuales europeas y estadounidenses se distinguen marcadamente de las trabajadoras mexicanas en que las primeras preferían la penetración oral a la vaginal, y en que ellas aceptaban prácticas para las cuales en la Ciudad de México había un rechazo notable. Por ejemplo, en el estudio de Green y colaboradores en Glasgow, Escocia, los servicios sexuales "convencionales" se definieron como sexo oral, masturbación y sexo vaginal. Por lo tanto, entre 56 trabajadoras sexuales de Glasgow, una media de $40 \%$ de los servicios que ellas definieron como "convencionales" fueron vaginales. Estas trabajadoras sexuales dijeron que los clientes pidieron servicios "no convencionales" frecuentemente, y ellas aceptaban darlos por el pago apropiado; sin embargo, a $87 \%$ les pidieron penetración anal y pocas dijeron que la aceptaron. ${ }^{35}$ Específicamente, entre las 68 trabajadoras sexuales estudiadas hubo un total de 764 casos de sexo vaginal, 1040 casos de sexo oral y 301 de masturbación en la semana anterior al estudio. ${ }^{36}$

En el estudio de McKeganey y Barnard, también en Glasgow, Escocia, el servicio más frecuente fue el sexo oral, y las mujeres dijeron preferir dar este servicio en lugar del sexo vaginal. Estas trabajadoras sexuales explicaron esta preferencia de tres maneras: percibían el sexo oral como menos riesgoso en términos de la transmisión del VIH; era más fácil puesto que daban los servicios en el coche del cliente, y decían que percibían el sexo oral como menos íntimo que la penetración vaginal. De 68 trabajadoras, en una noche refirieron 147 actos de sexo vaginal y 200 de sexo oral, 0 de sexo anal y 58 de masturbación. Aunque los clientes pidieron el sexo anal, ninguna de las trabajadoras dijo haber dado este servicio; esta petición fue una de las razones más frecuentes para rechazar a clientes. ${ }^{36}$

En el estudio relativamente pequeño (con 20 trabajadoras sexuales) realizado en Camden, Nueva Jersey, $6.1 \%$ de las participantes dijeron besar a clientes. El sexo oral fue la práctica más común (62.1\%) y todas las trabajadoras sexuales lo refirieron, mientras sólo $23.2 \%$ refirió la penetración vaginal, y se reportó un solo episodio de sexo anal. Freund y colaboradores proponen que el patrón de actividad sexual profesional (la cantidad de tiempo y el tipo de prácticas) de estas trabajadoras pudiera reflejar el que dan el servicio en los coches de los clientes. ${ }^{31}$ Asimismo, se observa un rechazo general de los besos con los clientes, igual que en México.

\section{Iniciación y disfrute sexual}

En cuanto a comparaciones entre la población femenina general de la Ciudad de México y las trabajadoras sexuales de nuestro estudio, hubo dos puntos que habría que discutir. En primer lugar, la edad media para la iniciación sexual entre las trabajadoras sexuales fue de 16.5 años (DS=2.752). En comparación, entre mujeres estudiadas a través de una encuesta representativa de viviendas de la Ciudad de México, realizada en 1988, fue de 17 años, ${ }^{39}$ mientras en un estudio realizado en 1993 la edad promedio fue de 18.3 años entre mujeres, ${ }^{40}$ y para el año 2000, la Encuesta Nacional de Salud había encontrado una edad promedio al inicio sexual de 14.9 años entre mujeres. ${ }^{41,42}$ Por otra parte, las trabajadoras sexuales incluidas en este estudio describieron su vida sexual privada como generalmente placentera para ellas, y mencionaron que incluye el orgasmo. Es interesante comparar esto con el disfrute sexual que refieren mujeres de la población general de la Ciudad de México del mismo nivel socioeconómico: en un estudio reciente, mujeres con estas características describían poco o ningún placer sexual en sus relaciones sexuales con sus parejas, y muchas no sabían de la existencia del orgasmo femenino. ${ }^{43}$

Con relación al disfrute sexual en los contactos sexuales con clientes, mientras nuestro estudio encontró un nivel extremadamente bajo de placer sexual (en el ámbito profesional) reportado por las trabajadoras, varios estudios en otros países han encontrado algo diferente. Por ejemplo, de las 62 trabajadoras sexuales colombianas que estudiaron Gallo y Alzate, 74\% refirieron tener orgasmos durante el sexo comercial, aunque el resto dijeron nunca tener orgasmos con clientes. ${ }^{33}$

En un estudio en Filadelfia ${ }^{44}$ enfocado específicamente en el nivel de disfrute sexual de 46 trabajadoras sexuales, 70\% de ellas dijeron disfrutar el sexo vaginal con clientes, $83 \%$ refirieron disfrutar recibir el sexo oral mientras 63\% disfrutaron darlo, y $43 \%$ dijo disfrutar el sexo anal por lo menos a veces. En todos los casos el disfrute sexual con la pareja privada fue mayor que con los clientes en este grupo de trabajadoras sexuales. Con las parejas personales, la mayoría de las participantes en el estudio (60.5\%) refirió siempre tener orgasmos, y el resto $(39.5 \%)$ dijo usualmente lo- 
grar el orgasmo con su pareja. Esto fue diferente con los clientes, ya que sólo $6.5 \%$ refirió siempre tener orgasmos; sin embargo, 57\% usualmente logró el orgasmo y $34.8 \%$ a veces, con los clientes. Sólo $19.6 \%$ dijo que rara vez y $17 \%$ que nunca tenían orgasmos con sus clientes. En esta población de estudio ninguna trabajadora sexual participante dijo que rechazaba por completo el sexo anal con los clientes, pero $15.8 \%$ sí lo hacía con sus parejas personales. El comportamiento con relación al sexo anal pareciera ser el opuesto entre nuestra población de estudio, donde el rechazo fue casi total para clientes pero con parejas personales se aceptaba más frecuentemente. Por otra parte, los autores del estudio sobre las trabajadoras sexuales de Filadelfia dicen que las mujeres incluidas en su población de estudio tuvieron un disfrute sexual en su vida privada más alto que lo reportado por el público en general, que posiblemente sea el caso entre nuestra población de estudio también.

Un estudio etnográfico que incluyó historias de vida con 35 trabajadoras sexuales que vivían en un pueblo ubicado en una carretera en Uganda encontró que las mujeres entrevistadas percibían el trabajo sexual como una manera de ganarse la vida y también algo que disfrutaban en el aspecto sexual. ${ }^{5}$ Mientras tanto, el estudio entre trabajadoras sexuales indonesias que vivían en Bali y trabajaban con clientes que eran turistas, encontró que las mujeres que percibían que los condones reducían su placer los usaban de manera menos consistente, lo cual implica que el placer juega un papel en sus actividades sexuales profesionales. ${ }^{34}$

\section{Uso del condón con clientes y parejas personales}

En nuestro estudio, las trabajadoras sexuales reportaron un alto nivel de uso del condón en sus encuentros sexuales con sus clientes. Según una reseña de varios artículos sobre comercio sexual hecha por Jackson y colaboradores, ${ }^{3}$ la mayoría de estos estudios norteamericanos también indican uso frecuente del condón por las trabajadoras sexuales en los contactos sexuales profesionales. Los autores de este artículo concluyen como nosotros que los condones funcionan como una barrera psicológica entre la trabajadora y el cliente, y que los condones representan una separación entre sus relaciones sexuales privadas y públicas. Un estudio cualitativo-cuantitativo entre un gran número de trabajadoras sexuales indonesias (en tres ciudades, $n=486, n=342, n=658)$ también encontró que usaron el condón para distanciarse en términos emocionales de los clientes. ${ }^{39}$
Otra reseña de diversos estudios existentes sobre el comercio sexual en todo el mundo, hecha por Estébanez y colaboradores, ${ }^{7}$ plantea la posibilidad de un incremento en el uso de condones entre trabajadoras sexuales. Sin embargo, los autores dicen que en varios lugares parece que las trabajadoras sexuales los usan más con clientes que con parejas personales, cosa que también observamos en nuestra población de estudio. Los mismos autores también reportan que el uso de lubricantes a base de aceite (que dañan el látex) podría estar asociado a infección con el VIH entre mujeres que no usan drogas intravenosas. Este hallazgo podría aplicarse a nuestra población, dado que las trabajadoras sexuales que estudiamos no usan drogas intravenosas $(0.27 \%, n=1)$ y algunas de ellas sí usan lubricantes a base de aceite.

De las trabajadoras sexuales de Glasgow del estudio de Green y colaboradores, $98 \%$ dijeron que siempre usaban condones durante la penetración vaginal y el sexo oral. ${ }^{35}$ Por otro lado, en el otro estudio realizado en Glasgow (por McKeganey y Barnard), de los 147 actos de sexo vaginal en la noche anterior, sólo en un caso una trabajadora dijo que no había usado condón; de 200 actos de sexo oral, sólo en dos casos dijeron no haber usado condón. Por otro lado, sólo dos de las 68 trabajadoras sexuales en este estudio dijeron que usaban condones con sus parejas personales. Según estos autores, parte de la razón fue para distinguir el sexo comercial del personal. ${ }^{36}$

En el estudio de Freund y colaboradores en la ciudad de Camden, Nueva Jersey, (EUA) 74\% de las trabajadoras sexuales dijeron usar condones en el último contacto sexual y seis (de 20) participantes dijeron siempre usar condones con sus clientes. ${ }^{37}$ En general, estos hallazgos se parecen a los nuestros en que un alto porcentaje de trabajadoras sexuales dijeron usar condones con sus clientes. La falta de uso del condón con parejas personales y las razones que motivan este comportamiento riesgoso también son similares.

Los estudios que arrojaron resultados algo diferentes de los nuestros en cuanto al uso del condón fueron los dos estudios realizados por Wilson y colaboradores en Bulawayo y Harare, Zimbabwe. Por ejemplo, las trabajadoras sexuales en Bulawayo dijeron haber usado condones en sólo $39.3 \%$ de los contactos sexuales con clientes en la semana pasada. ${ }^{32}$ En Harare, de 596 contactos sexuales, las trabajadoras sexuales refirieron que en 430 usaron condón. ${ }^{17}$

El estudio hecho entre 80 trabajadoras sexuales de Bali encontró que mientras $90 \%$ de ellas reportaron uso del condón con clientes, solamente 5\% lo reportó con las parejas íntimas en la última semana. ${ }^{34}$ En otro estudio en Indonesia, con 1486 trabajadoras sexuales, ellas 
reportaron no pedir el uso del condón a sus clientes regulares que conocían y con quienes tenían una relación de amistad; ni siquiera pensaban en usar condones con sus parejas privadas (novios). ${ }^{45}$ Un estudio etnográfico con 30 trabajadoras sexuales, también en Indonesia, encontró que tampoco usaban condones con sus clientes regulares ni con sus parejas privadas. ${ }^{22} \mathrm{Un}$ estudio cualitativo en Australia encontró que las 12 trabajadoras sexuales entrevistadas muy rara vez usaban condones con sus parejas personales, mientras otro estudio en Australia, en este caso cuantitativo, reportó lo mismo. ${ }^{46,47}$

En Tanzania, dos estudios etnográficos entre trabajadoras sexuales ( $n=128, n=50$ ) encontraron que el uso del condón era común con clientes ocasionales pero casi inexistente entre clientes con los cuales las mujeres sostenían una relación emocional y económica a largo plazo. Sin embargo, en este caso la razón por la cual no usaban condones con las parejas de larga duración era principalmente económica y la cuestión emocional quedaba en segundo término. ${ }^{48}$ Un estudio entre 600 trabajadoras sexuales en tres ciudades brasileñas también encontró que pocas trabajadoras sexuales usaban condones con sus parejas personales, pero en este caso la razón fue que temían violencia por parte de sus parejas si insistían en el uso del condón con ellos. ${ }^{49}$

\section{Limitaciones}

Aunque nosotros encontramos un alto nivel de uso del condón entre la población de estudio, no podemos decir que no exista ningún tipo de duda sobre este hallazgo. En sus respuestas al cuestionario y durante las discusiones grupales, las participantes en el estudio mantenían que siempre usaban el condón con sus clientes. Sin embargo, contradicciones en su discurso durante las entrevistas e información indirecta (de la observación) indican que el nivel de uso podría no ser tan elevado como ellas lo describen. Por ejemplo, en un estudio etnográfico sobre trabajadoras sexuales de sitios de calle de nivel medio-alto de la Ciudad de México, Lamas concluye que el uso real del condón no es tan frecuente como se informa, aunque las participantes dicen usar condones constantemente en los cuestionarios y las entrevistas. ${ }^{44}$ Un artículo escrito por varios miembros del entonces CONASIDA también sugiere que el nivel de uso del condón entre trabajadoras sexuales es menor al reportado, una conclusión basada en parte en los niveles de otras infecciones de transmisión sexual que se han encontrado en esta población. ${ }^{11}$ Por otra parte, en general, las limitaciones de este estudio son las de los estudios transversales, que no pueden dar explicaciones causales.

\section{Conclusiones y recomendaciones}

Nuestro análisis nos lleva a concluir que las trabajadoras sexuales que laboran en sitios de calle en la Ciudad de México se beneficiarían de programas de promoción del sexo más seguro si tomaran en cuenta tanto su vida profesional como la privada. En cuanto a sus vidas privadas, las estrategias serían iguales a las que podrían servir para todas las mujeres, incluyendo:

- promover el uso del condón en relaciones a largo plazo;

- la desestigmatización o domesticación del condón para romper su asociación exclusiva con el sexo comercial;

- el acceso fácil y barato a servicios de salud (que tuvieran además calidad y calidez) para el tratamiento y la prevención de otras infecciones de transmisión sexual;

- la promoción del condón entre varones, para que su uso no implique una posible reacción de violencia o de abandono emocional y económico de la mujer por parte del hombre con el cual tiene una relación a largo plazo. ${ }^{8,48,49}$

Las estrategias para promover prácticas seguras en su trabajo podrían incluir las siguientes:

- hacer disponibles (y baratos) en los lugares de trabajo los condones y los lubricantes a base de agua;

- la promoción del sexo más seguro entre las trabajadoras sexuales a través de la educación de pares (es decir, impartida por trabajadoras sexuales entrenadas para educar a sus colegas);

- la capacitación de las trabajadoras sexuales para que se sientan eficaces en el uso y la negociación del condón;

- la promoción de la formulación de políticas de uso constante del condón por parte de administradores de los sitios de trabajo sexual;

- esfuerzos de educación y erotización de condones enfocados en los clientes. ${ }^{6,13,14,22,34,48}$

Podría ser importante para la efectividad de estas estrategias el que se dieran dentro de un contexto en el cual las trabajadoras sexuales tuvieran oportunidades para que entre sí pudieran compartir experiencias y apoyarse en la adopción de nuevos comportamientos más seguros. Las mujeres que participaron en los grupos focales para este estudio dijeron que nunca antes habían podido hablar de su trabajo, pero cuando empezaron a hacerlo, expresaron su deseo de tener más oportunidades de compartir esta parte de su vida. 
Oportunidades de este tipo, para hablar y compartir, pueden ser en sí un paso hacia la superación de normas sociales que le dan a la mujer menos poder en la negociación del sexo más seguro. ${ }^{8}$ Además, pueden crear una mayor solidaridad entre trabajadoras sexuales ${ }_{1}^{17}$ y crear un foro para la discusión de posibles maneras de eliminar barreras al sexo más seguro. ${ }^{48,49}$

\section{A gradecimientos}

La colaboración de personal del entonces llamado Consejo Nacional para la prevención y el control del SIDA (CONASIDA) fue indispensable para la realización de este estudio, en especial la de Laura Elena de Caso, Víctor Ortiz y la doctora Patricia Uribe.

\section{Referencias}

1. Herrera C, C ampero L. La vulnerabilidad e invisibilidad de las mujeres ante el VIH/SIDA: constantes y cambios en el tema. Salud Publica Mex 2002;44(6):554-565.

2. A maro H. Love, sex, and power. Considering women's realities in HIV prevention. Am Psychol 1995;50(6):437-447.

3. Jackson L, Highcrest A, C oates R.Varied potential risks of HIV infection among prostitutes. Soc Sci Med 1992;35(3):281-286.

4. Sacks V.W omen and AID S:An analysis of media misrepresentations. Soc Sci Med 1996;42(1):59-73.

5. Gysels M, Pool R, N nalusiba B.W omen who sell sex in a U gandan trading town: Life histories, survival strategies and risk. Soc Sci Med 2002;54(1):179-192.

6. W ojcicki JM, Malala J. Condom use, power and HIV/AIDS risk: Sexworkers bargain for survival in Hillbrow/Joubert Park/Berea, Johannesburg. Soc Sci Med 2001;53(1):99-121.

7. Estébanez P, Fitch K, Nájera R. HIV and female sex workers. Bull W orld Health $O$ rgan 1993;71(3/4):397-412.

8. Rao G upta G,W eiss E.W omen's lives and sex: Implications for AID S prevention. Conference on International Perspectives in Sex ResearchARHN W orking Group on Sexual Behavior Research; 1993 A pril 22-25: Río de Janeiro, Brazil.

9. Padian N . Editorial Review. Prostitute women and AIDS: Epidemiology. AIDS 1988;2:413-419.

10. Del Río C, Sepúlveda J.AIDS in Mexico: Lessons learned and implications for developing countries. AID S 2002;16(11):1445-1457. 11. U ribe-Zúñiga $\mathrm{P}$, H ernández-Tepichín $\mathrm{G}$, D el Río-C hiriboga C, 0 rtiz V. Prostitución y SIDA en la Ciudad de México. Salud Publica Mex 1995;37:592-601

12. Uribe-Salas F, Hernández-A vila M, C onde-G onzález C, Cruz-Valdez A, Juárez-Figueroa L,Allen $B$ et al. Low prevalence of HIV infection and sexually transmitted disease among female commercial sex workers in Mexico City.Am J Public Health 1997;6:1012-1015.

13. Liguori AL. Relaciones de género y apoderamiento femenino. En: Hernández Avila M,Vandale Toney S, Liguori AL, ed. Enfoques de investigación sobre VIH/SIDA en salud reproductiva. Cuernavaca: Instituto N acional de Salud Pública, 1995:81-95.
14. Campbell C. Selling sex in the time of AIDS:The psycho-social context of condom use by sex workers on a Southern African mine. Soc Sci Med 2000;50(4):479-494

15. A samoah-Adu C, Khonde N, Avorkliah M, BekoeV,Alary M, Mondor et al. HIV infection among sex workers in Accra: $N$ eed to target new recruits entering the trade. J Acquir Immune D efic Syndr 2001;28(4):358-366.

16. Joesoef MR, Kio D, Linnan M, Kamboji A, Barakbah Y, Idajadi A. Determinants of condom use inf female sex workers in Surabaya, Indonesia. Int J ST D AID S 2000;11(4):262-265.

17. W ilson D, Chiroro P, Lavelle S, Mutero C. Sex worker, client sex behaviour and condom use in $\mathrm{H}$ arare, Zimbabwe.AIDS Care 1989:1(3):269-280.

18. Lian W M, Chan R, W ee S. Sex workers' perspectives on condom use for oral sex with clients:A qualitative study. Health Educ Behav 2000;27(4):502-516.

19. G hys PD, Jenkins C, Pisani E. HIV surveillance among female sex workers.AIDS 2001;15(Suppl 3):S33-S40.

20.Allen B, Arana ME, O rtiz-A guirre V, D e C aso LE. Identidad, disociación corporal y salud sexual entre mujeres que hacen trabajo sexual en la ciudad de México. Géneros, 2002;10(28):45-54.

21.W eeks J. History, desire, and identities. Conference on International Perspectives in Sex Research-ARHN W orking Group on Sexual Behavior Research; 1993 A pril 22-25 Río de Janeiro, Brazil. 22. Sedyaningsih-Mamahit ER. Female commercial sex workers in Kramat Tunggak, Jakarta, Indonesia. Soc Sci Med 1999;49(8):1101-1114. 23. Bronfman M, G uiscafré $H, C$ astro V, C astro R, Gutiérrez G. La metodología y análisis de las características socio-económicas de la muestra. Arch Invest Med 1988;19:351-360.

24. U ribe F, Hernández-Avila $M$, C onde $C$, Juárez L, U ribe $P$, D el Río $C$ et al. Estudio de prevalencia de ETS en MTSC de la Ciudad de México basado en la construcción de un marco muestral. Gac Med Mex 1994;130(supl.1):97-98.

25. Pyett PM,W arr DJ.Vulnerability on the streets: Female sex workers and HIV risk.AIDS Care 1997;9(5):539-547.

26. G laser B, Strauss a. The discovery of grounded theory. C hicaco (IL): Aldine, 1967.

27. Glaser B. Basics of grounded theory analysis. Mill valley (CA):

Sociology Press, 1992.

28. Stern PN , Pyles S. U sing grounded theory metodology to study women's culturally-based decisions about health. En: stern PN , Ed. W omen, health and culture. Washington, DC: Hemisphere, 1986:1-24. 29. Keddy B, Sims L, Stern PN . G rounded theory as a feminist research methodology. J Adv N urs 1996;23:448-453.

30. West J. Feminist grounded theory: An exploration of the congruency and tensions between two traditions in knowledge discovery. Q ual Health Res 1996;5:125-137.

31. Lamas M. El fulgor de la noche: algunos aspectos de la prostitución callejera en la ciudad de México. D ebate Feminista 1993:4(8):103-136. 32.W ilson D, Sibanda B, Mboyi L, Msimanga S, D ube G.A pilot study for an HIV prevention programme among commercial sex workers in Bulawayo, Zimbabwe. Soc Sci Med 1990;31(5):609-618.

33. De Gallo MT,Alzate H. Borthel prostitution in Colombia. Arch of Sexual Behav 1976;5(1):1-7.

34. Thorpe L, Ford K, Fajans P,W irawan DN . Correlates of condom use among female prostitutes and tourist clients in Bali, Indonesia.AIDS Care 1997:9(2):181-197.

35. Green ST, Goldberg DJ, Christie PR, Frischer M, Thomson A, C arr SV et al. Female streetworker-prostitutes in Glasgow:A descriptive study of their lifestyle.AIDS Care 1993; 5(3):321-335.

36. McKeganey N, Barnard M. Selling sex: Female street prostitution and HIV risk behaviour in G lasgow. AID S Care 1992;4(4)395-407. 
37. Freund M, Leonard T, Lee N . Sexual behavior of resident street prostitutes with their clients in Camden, $\mathrm{N}$ ew Jersey. Sex Res 1989;26(4):460-478.

38. Pickering H, Todd J, D unn D, Pepin J,W Ilkins A. Prostitutes and their clients: A Gambian survey. Soc Sci Med 1992;34(1):75-88.

39. Morris L, N úñez L, Monroy de Velasco A, Bailey P, Cárdenas C, $W$ hatley $A$. Sexual experience and contraceptive use among young adults in Mexico City. International Fam Plann Perspec 1988;14(1):147-152.

40. G arcía-Baltazar J, Figueroa-Perea JG, Reyes-Zapata H, Brindis C, Pérez-Palacios G. C aracterísticas reproductivas de adolescentes y jóvenes en la Ciudad de México. Salud Publica Mex 1993;35(6):682-691. 41. Pérez-Islas JA . Encuesta N acional de la Juventud 2000. México, DF: Secretaría de Educación Publica / Instituto Mexicano de la Juventud, 2002. 42. G ayet C, Juárez F, Magis C, Pedrosa L. U so de condón y primera relación sexual en adolescentes.Trabajo \#193IN VS, presentado al VIII Congreso Nacional deVIH/SIDA: 2002 diciembre 4,5 y 6;Veracruz, Veracruz, México

43. Zubiliaga-Rodríguez AC. Sexualidad de mujeres de colonias populares del Distrito Federal, México. Ponencia al XX Congreso
Latinoamericano de Sociología:A mérica Latina y El Caribe, perspectivas de su reconstrucción; México, D F, México, 1995 octubre 2-6.

44. Savitz L, Rosen L.The sexuality of prostitutes: Sexual enjoyment reported by streetwalkers. Sex Res 1988;24:200-208.

45.W olffers I, Subarniati-Triyoga R, Basuki E,Yudhi D, D evilléW, Hargono R. Pacar and Tamu: Indonesian women sex workers' relationships with men. Culture Health Sexuality 1999;1(1):39-53.

46. W addell C. HIV and the social world of female commercial sex workers. Med Anthropol Q 1996;10(1):75-82.

47. Pyett PM, Haste BR, Snow J. Risk practices for HIV infection and other STD s amongst female prostitutes working in legalized borthels. AIDS Care 1996:8(1):85-94.

48. 0 utwater A, N kya L, Lwihula G, 0 'C onnor P, Leshabari M, $N$ guma J et al. Patterns of partnership and condom use in two communities of female sex workers in Tanzania. J Assoc N urses A IDS C are 2000;11(4):46-54.

49. Lurie P, Fernández ME, Hughes V, A révalo El, Hudes ES, Reingold $A$ et al. Socioeconomic status and risk of HIV-1, syphilis and hepatitis B infection among sex workers in Sao Paulo state, Brazil.AIDS 1995: 9 (Suppl 1):S31-S37. 